\title{
ZigBee Radio Frequency (RF) Performance on Raspberry Pi 3 for Internet of Things (IoT) based Blood Pressure Sensors Monitoring
}

\author{
Puput Dani Prasetyo Adi ${ }^{1}$, Akio Kitagawa ${ }^{2}$ \\ Micro Electronics Research Laboratory (MeRL), Kanazawa University, Kanazawa, Ishikawa, Japan
}

\begin{abstract}
Wireless Sensor Network has grown rapidly, e.g. using the Zigbee RF module and combined with the Raspberry Pi 3 , a reason at this research is building a Wireless Sensor Network (WSN). this research discusses how sensor nodes work well and how Quality of Service (QoS) from the Sensor node being analyzed and the role of Raspberry $\mathrm{Pi} 3$ as an internet gateway will sending a blood pressure data to the database and displayed in real-time on the internet, from this research it is expected that patients can check the blood pressure from home and don't need to the Hospital even data can be quickly and accurately received by Hospital Officers, doctors, and medical personnel. the purpose of this research is make a prototype to providing a blood pressure $(\mathrm{mmHg})$ real-time data from systolic and diastolic data patient's that determine patients suffering from symptoms of certain diseases, i.e, anemia, symptoms of hypertension and even more chronic diseases. this research discusses how sensor nodes work well and how Quality of Service (QoS) from the Sensor node being analyzed and the role of Raspberry $\mathrm{Pi} 3$ as an internet gateway will sending a blood pressure data to the database and displayed in real-time on the internet. Furthermore, Zigbee has the task of sending Blood pressure $(\mathrm{mmHg})$ data in real-time to the database and then sent to the internet from Zigbee end-device communication to ZigBee coordinator. Zigbee communication at a distance of 5 meters, RSSI simulations show a value of $-29 \mathrm{dBm}$ and the experiment shows a value of $-40 \mathrm{dBm}$, at a distance of $100 \mathrm{~m}$, RSSI shows a value of $\mathbf{- 5 5} \mathrm{dBm}$ (simulation) and $\mathbf{- 8 6} \mathrm{dBm}$ (experiment).
\end{abstract}

\section{Keywords-Zigbee; Raspberry Pi 3; IoT; blood pressure}

\section{INTRODUCTION}

At present, the Internet of Things (IoT) is growing rapidly, with the development of various platforms and security systems i.e, the ZigBee RF module, WiFi Module, Bluetooth (BLE) module, and Micro-electromechanical system (MEMS). the Internet of Things (IoT) processing data on the internet, i.e, sensor data. IoT allows automation and convenience so that data automatically will be sent to the internet and can be viewed in real-time. i.e, on a Personal Computer Platform, a tablet and a Smartphone has an internet connection. WiFi modules are compatible with Internet of Things Sensors such as Fingerprint, references [1] investigated WiFi Positioning based on fingerprinting and Quality of Service (QoS) and Receive Signal Strength Indicator (RSSI) from sending sensor data using the Positioning of WiFi module. With IoT Technology, human life becomes easier and more flexible, especially in this research, IoT in the health sector i.e, monitoring Blood Pressure using a ZigBee device as data senders from the Sensor Node to the Coordinator node located on the Raspberry Pi 3 as an internet gateway.

However, IoT devices are faced with the problem of attacks from malicious software [2] so a Security tools method is needed that can protect IoT devices to continue working stably. accordingly, The latest version of Bluetooth devices, BLE is a device with Low Energy that guarantees IoT device Life Time. Bluetooth devices like RN-42 [3] are needed as devices that can communicate Master-Slave, this Bluetooth type can be combined with Internet Gateway with the same Raspberry Pi 3 in the research conducted in this paper. In the study [4] examined about Collaborative, Seamless and Adaptive Sentinel for the Internet of Things (COSMOS) Apps to protect the smart environment from cyber threats. In addition to using the Zigbee RF Module, Bluetooth standards such as the RN-42, or Bluetooth Low Energy (BLE), sending sensor data can use the Global System for Mobile Communication (GSM) device, which includes a Wireless communication system with a wide range of research sensors, namely ultrasonic sensors using GSM SIM900A [5] to smartphone or mobile devices, the similarity of this research is the sensor data transmission system through the Wirelessbased System platform. Apart from GSM other data sender technology devices are Radio Frequency Identifier (RFID), 3G, UMTS, WiFi, BLE, infrared and Zigbee which are distinguished by the Object Abstraction Layer.

Accordingly from the data share of dominant IoT application project by 2025 , the biggest potential economic impact of sized IoT Applications are Health Care $41 \%$, manufacturing 33\%, Electricity 7\%, Urban infrastructure 4\%, Security 4\%, Resource Extraction 4\%, Agriculture $4 \%$, Vehicle $2 \%$ and Retail 1\%. [6]. therefore, this is the biggest reason this paper was created is to develop the role of IoT in the field of Health Care using Zigbee Performance Pipe which is rarely used as an IoT support device. e.g. on reference [7] examined the application of IoT to optimize the performance of vehicle tracking in cloud servers. Device Healthcare support platforms such as e-health can be combined with IoT and can be analyzed for the level of security, reliability, and architecture they have [8], moreover, e-health is very compatible with many sensors such as SPo2, blood pressure, and pulse sensors. In another study, an approach to Pacemaker Pulse detection [9] was carried out in certain patients who needed Pacemaker, this sensor was able to detect Pacemaker surges up to $12 \mathrm{~cm}$ from Pacemaker leads. Dimiter V. Dimitrov, MD, Ph.D. [10] in his research tried to see and 
analyze complex data / Big Data on IoT in the field of Healthcare, which consisted of Clinical Data, EHR Data, Molecular omics data, and Wearables data that entered the Repository data, furthermore, a Big Data The warehouse that was then analyzed, happened Dimensionality Reduction, segmentation and ended with Big Data with the number of Billion in the form of Medical Apps developers.

\section{RELATED STUDIES}

Giorgio Biagetti, Paolo Crippa, Laura Falaschetti and Claudio Turchetti [11], in this research installing a device called surface electromyographic (sEMG) and an accelerometer on the arm of a person doing fitness then sEMG data and accelerometer sent with Wireless Sensor Network devices, then classified according to level Fusion, consequently, the accuracy of the results of the SEMG data transmission and accelerometer is $82.6 \%$ of all types of styles during fitness activities, pada research ini data yang dihasilkan adalah sEMG data and accelerometer, in this research the data produced is sEMG data and accelerometer, in this research can be developed using Kalman Filtering and Learning Algorithms. The position of the sensor is like this research, which is on the wrist that takes the movement of the arteries.

Muhammad Niswar, Amil Ahmad Ilham, Elyas Palantei, Rhiza S. Sadjad, Andani Ahmad, Ansar Suyuti, Indrabayu, Zaenab Muslimin, Tadjuddin Waris, Puput Dani Prasetyo Adi [12], in this research using Zigbee as an RF Module to transmit pulse data patients in different distances, in this research, pulse data is sent from 5 sensor nodes simultaneously to one ZigBee node coordinator. Data received by ZigBee experiences Packet Loss when sending sensor node data to 4 sensor nodes, consequently, that $80 \%$ of the pulse data is received by the Coordinator node, this occurs because of the bottleneck factor in the ZigBee coordinator, bottleneck due to the multiplexing factor that occurs in Zigbee coordinator. accordingly, This is due to simultaneous data transmission by 5 sensor nodes, data can be received in stages but a decrease factor in Quality of Service (QoS), Receiver Signal Strength Indicator and Pathline when sending data bits per second (bps). Packet loss can be calculated from the reduction in bits sent by the receiver. In this research Radio Frequency devices used are the same as research [12], the difference is in the type or type of XBee e.g, XBee S2C and XBee S1 Pro.

Muhammad Anwar, Abdul Hanan Abdullah, Ayman Altameem, Kashif Naseer Qureshi, Farhan Masud, Muhammad Faheem, Yue Cao, and Rupak Kharel [13], in their research tried to implement the Wireless Body Area Network (WBAN) on the patient's body and analyze its routing system using the energy-aware link efficient routing approach (ELR-W), therefore, the goal is to save energy from the Wireless Body Area Network (WBAN). accordingly, in Wireless Sensor Network (WSN), WBAN, M2M Communication, it is very important for energy efficiency factors, i.e, batteries used, so the use of dynamic Power Supply such as PMFC is important, Plant-Microbial Fuel Cell (PMFC) as an energy source is especially appropriate in designing indoor systems or Outdoor environments [14], In this research, it refers to WBAN technology [14], by developing a Blood Pressure sensor with a light type that is convenient to be used by Patients.

Moh. Khalid Hasan, Md. Shahjalal, Mostafa Zaman Chowdhury and Yeong Min Jang [15], in this research using Bluetooth Low Energy-based Wireless Sensor Network devices and the board used, is e-Health connected to the sensor electrocardiogram (ECG) using the hybrid OCC / BLE System. furthermore, From this research, a comparison of Quality of Service (QoS) in the OCC, BLE and Hybrid Schemes was produced. ECG has more complex output than Blood Pressure, ECG placement is also in patients 'chest and sensor placement which is at several points on patients' chest, while blood pressure only takes on the base of the wrist or wrist tip.

Gaël Loubet, Alexandru Takacs, Ethan Gardner, Andrea De Luca, Florin Udrea and Daniela Dragomirescu [16], using LoRa as a Wireless Sensor Network device to monitor patient health, LoRAWAN is Vehicular-based communication [17]. This LoR works on the $868 \mathrm{MHz}$ ISM frequency radio. in this research the communication system built is Mesh Network with an approach to battery-free LoRaWAN sensing and communication nodes. accordingly, The power density of the electromagnetic waves is higher than $0.5 \mu \mathrm{W} / \mathrm{cm} 2$. So the Energy Consumption (EC) factor is important to be reduced to get a Long Life factor and stabilize the Packet Delivery Ratio (PDR) factor on reference [18] by evaluating root mean square error and deep learning method can produce $98 \%$ accuracy on PDR and EC Predictions. The use of LoRAWAN RF will be a good comparison with ZigBee RF in terms of QoS and Ability at long distances and hilly locations. In future research, it is necessary to compare and analyze the two types of RF Modules to send sensor data to Health monitoring.

Adolfo Di Serio, John Buckley, John Barton, Robert Newberry, Matthew Rodencal, Gary Dunlop 3 and Brendan O'Flynn in reference study [19], use the Zigbee to sending a heart rate data (BPM) and arterial Oxygen saturation ( $\mathrm{SpO} 2)$ data. ZigBee works on the $2.4 \mathrm{GHz}$ frequency, but several other Wireless Sensor Network (WSN) devices have lower frequencies, for example the ISM Band $915 \mathrm{MHz}$ frequency. The $915 \mathrm{MHz}$ frequency shows a $10 \mathrm{~dB}$ return loss bandwidth of $55 \mathrm{MHz}$, with a gain value of $-2.37 \mathrm{~dB}$ in free-space and $6.1 \mathrm{dBi}$ on-body. In the references [20] using IEEE 802.15.4 Protocol simulation using Q-Learning to improve the performance of MAC Protocol and obtained a comparison of values from average latency, average backoff, channel access ratio, and transmission success ratio. The board used is the STM32F769 board and STM32L486 board which has a Cortex M7 processor (216 MHz and $120 \mathrm{MHz}$ ) and Cortex M4 (80 MHz). The SpO2 sensor is precise when combined with a Blood Pressure sensor in this Research. Complex data will make the patient's examination more detailed and accurate.

\section{MATERIAL AND METHOD}

\section{A. Block Diagram that Represents Research as a Whole}

Overall this research system is shown in Fig. 1. There are 3 parts shown in the dashed line showing three parts to be analyzed, the first part is the ZigBee RF Module connection 
on the End Device and ZigBee RF Module on the Raspberry Pi 3. This section can be analyzed from Quality of Service (QoS) which includes the Power Receiver (PRx) and Receiver Signal Strength Indicator (RSSI). The construction of a star, tree, and mesh network is in the first part of the analysis, furthermore, then in the second analysis is Python programming and its connection using PuTTY and Web Server using XAMPP or WAMP followed by Website-based programming languages using HTML, PHP and Javascript or JSON can emerge Blood Pressure charts in real time in various platforms, at this stage, access to the domain is needed in order to be able to connect to the internet in realtime which is depicted at the 3rd analysis stage. furthermore at this research will be using the tool editor in HTML, Javascript or JSON using Dreamweaver Creative Cloud (CC) Software.

\section{B. LM358N Operational Amplifier}

LM358N Op-Amp is a Low Power IC, easy to use on a dual channel op-amp. The function of an LM358N Operational Amplifier is a signal amplifier in AC and DC currents and as a high input impedance differentiation amplifier and a low impedance output amplifier. accordingly, IC LM358N Operational Amplifier can handle 3-32 Volt DC supply and Source up to $20 \mathrm{~mA}$ per channel. In Fig. 1 Schematic of LM358 N Op-amp shows Voltage Controlled Oscillator (VCO). In this case, the LM358N Operational Amplifier is applied to the sensor node, in Fig. 2.

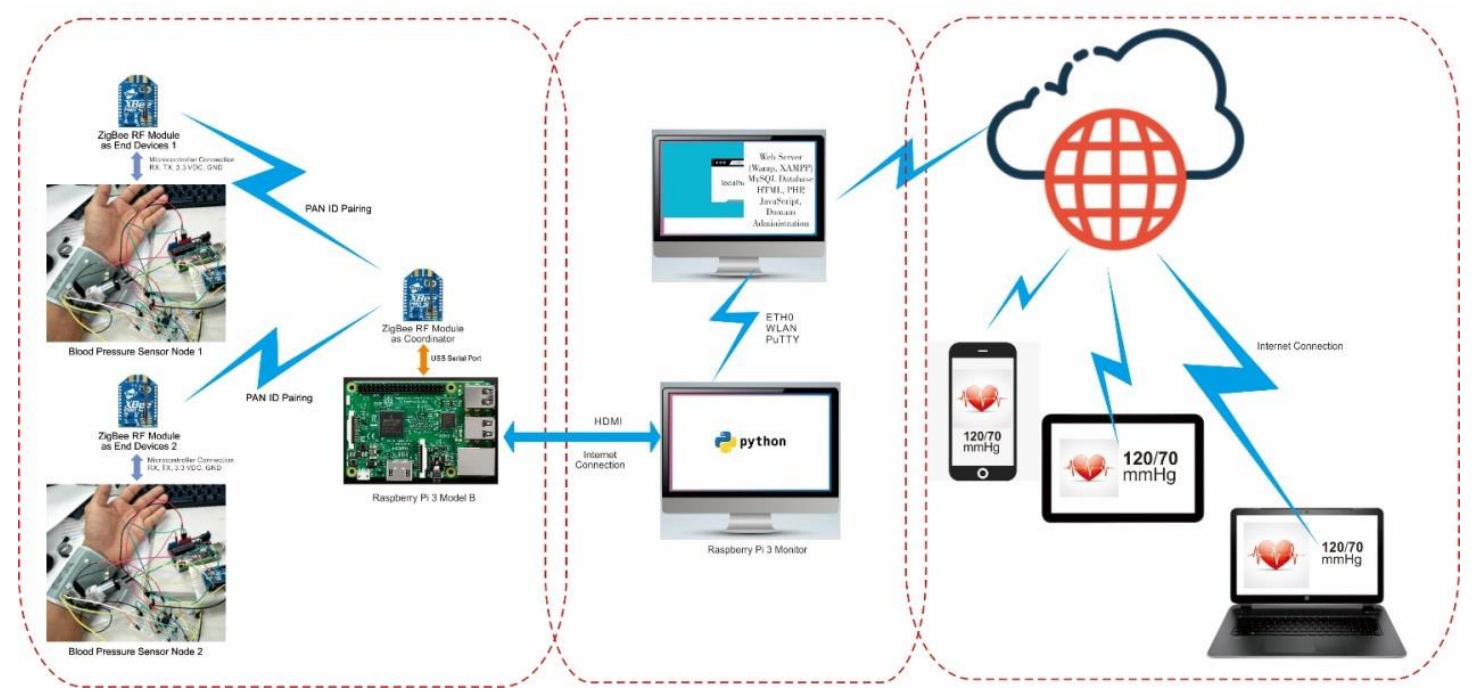

Fig. 1. Block Diagram that Represents Research as a Whole.

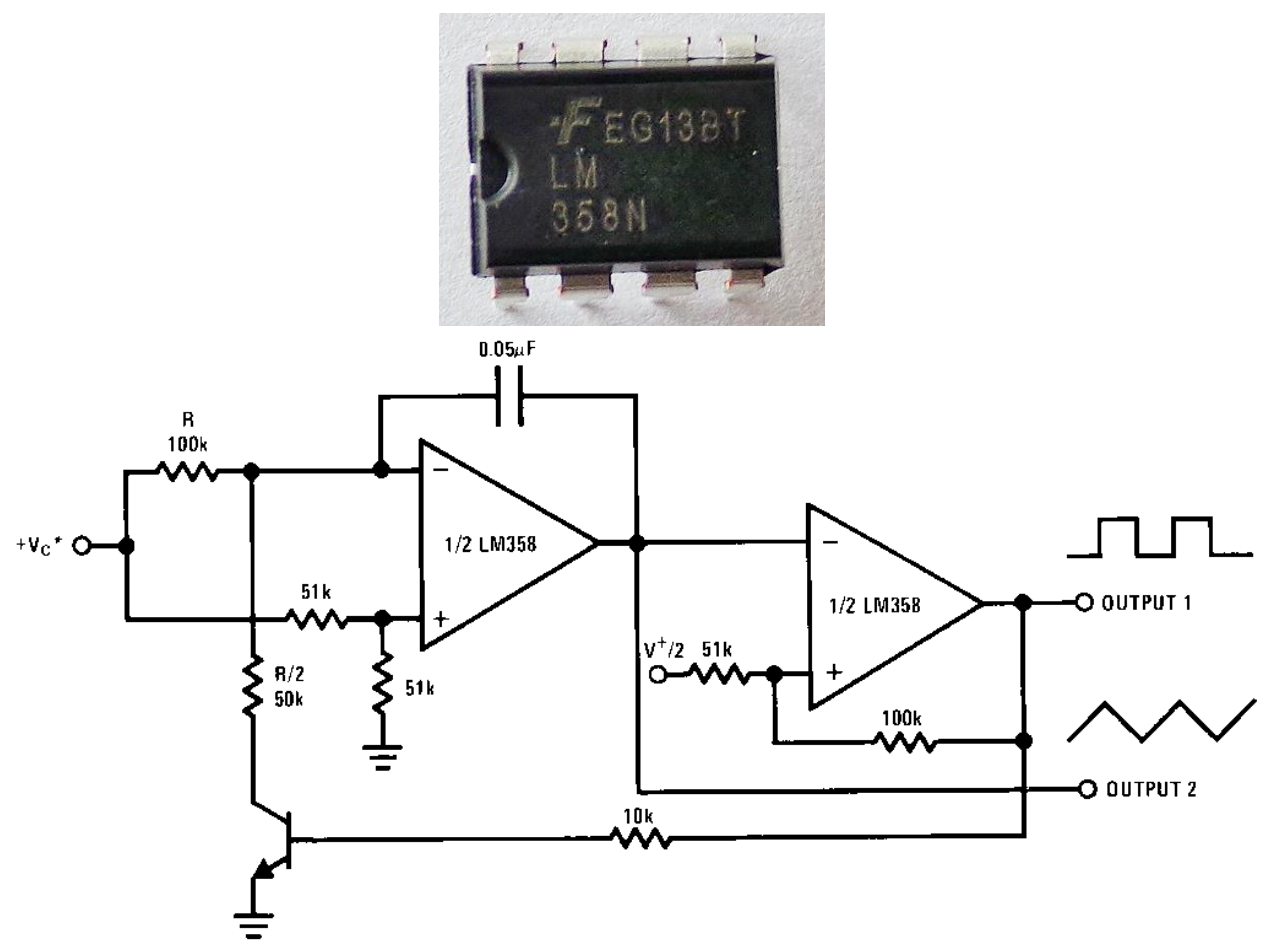

Fig. 2. IC and Schematic of LM358N Op-Amp. 


\section{Blood Pressure Sensor}

Accordingly, on this research, The pressure sensor used is the MPS20N0040D-S type, this is a kind of solid pressure sensor, using MEMS technology, high reliability, and low cost. The pressure range is $0-5.8$ Psi (40 kpa), the electricity supply is 5 volts DC and Constant Current is $1 \mathrm{~mA}$. the input impedance of 4-6 $\Omega$. bias voltage $\pm 25 \mathrm{mV}$, full-scale output voltage 50-100 mV. In Fig. 3 shows the module of the Pressure Sensor used in this research. furthermore, Fig. 4 is a Pressure Sensor Block Type MPS20N0040D-S used in this research.
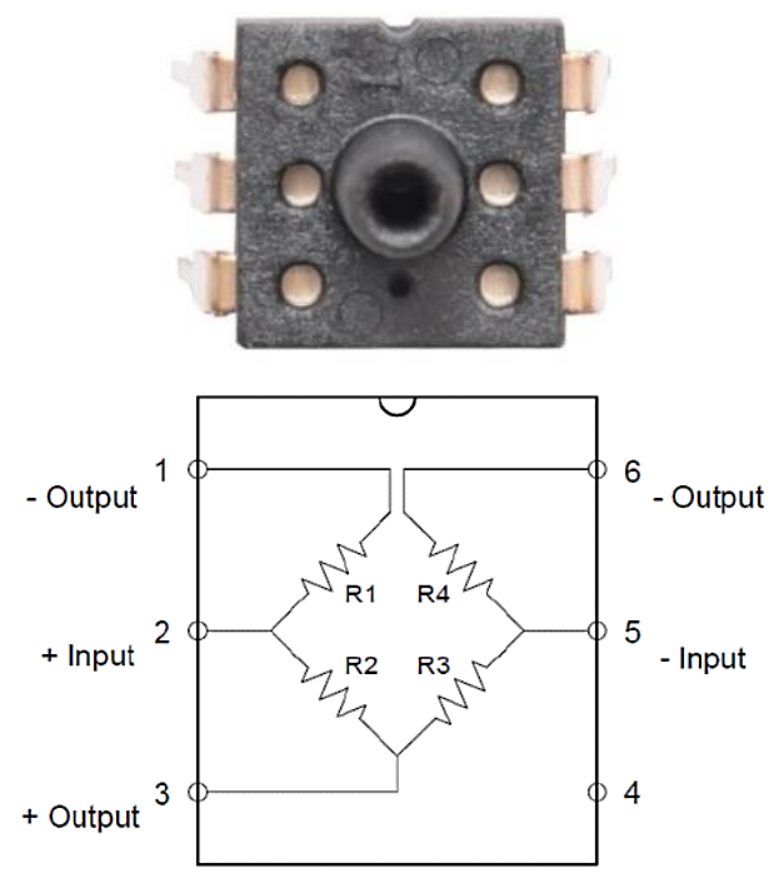

Fig. 3. Pressure Sensor.
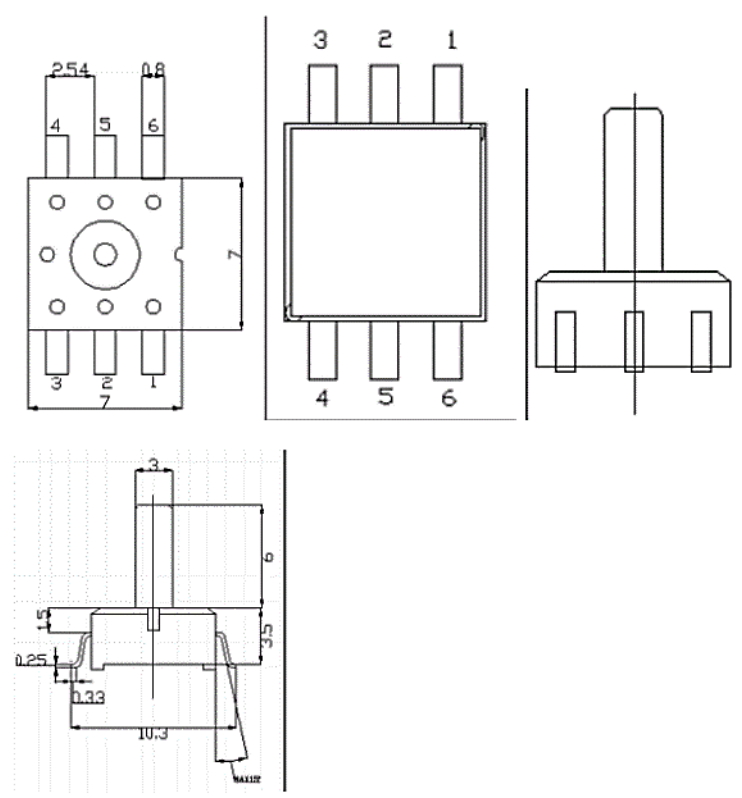

Fig. 4. Block Diagram and Dimensions of Pressure Sensors.
In Fig. 6. It shows that the classification of blood pressure is divided into three, i.e, Ideal blood pressure, pre-high blood pressure, and high blood pressure. And there are two types of terms used to measure the 3 classifications e.g, Systolic (Top number) and Diastolic (Bottom Number). furthermore, Data in Fig. 6. In accordance with data Systolic and diastolic is as follows, e.g, 150/80 $\mathrm{mmHg}$.

In Fig. 5. shows a Blood Pressure sensor diagram, in this diagram it will be developed using the MCU with Micro Arduino or Nanoduino so that the light version is convenient to be used by Patient.

The heart is the most important part of the body that is responsible for pumping blood throughout the body. accordingly, from the results of the examination can be concluded that the patient suffers from a particular disease, such as symptoms of stroke or heart disease. It is necessary to know the unit to state the amount of blood pressure is Millimeters of Mercury (Hydrargyrum) and then abbreviated $\mathrm{mmHg}$. While KiloPascals $(\mathrm{kPa})$ is a unit based on Standard International (SI) to express the amount of Pressure value, so to change $\mathrm{mmHg}$ to $\mathrm{Kpa}$ is $1 \mathrm{mmHg}$ equal 133,322 Pascals (Pa) then $1 \mathrm{kPa}$ equal 1000 Pascals $(\mathrm{Pa})$ then $\mathrm{mmHg}$ Value $\mathrm{x}$ $133,322 \mathrm{~Pa}$ equal $\mathrm{kPa}$ value $\mathrm{x} 1000 \mathrm{~Pa}$ or $\mathrm{mmHg}$ value equal $\mathrm{kPa}$ value $\mathrm{x}$ 7.50062. while Psi stands for Pound Per square inch (Psi), Psi is used to express the value of pressure other than using $\mathrm{kPa}, 1$ Psi equal 6.89475729 kilopascals.

In Fig. 5 a Blood Pressure Sensor block diagram contains several important components i.e, MCU is part of the Data Controller and Processor, can also be called a Microcontroller, in this research used Arduino Micro Microcontroller. Then the Output section is LCD, the LCD used for research development is an $8 \times 2$ bit LCD. With an actuator, a DC motor which is assigned to provide pressure in the form of air and a Pressure sensor component (Fig. 5) are connected to the amplifier circuit and Band Pass Filter. The amplifier circuit with the Band Pass Filter functions as a filter for analog frequencies. In detail, the Union body of the blood pressure sensor can be seen in Fig. 7.

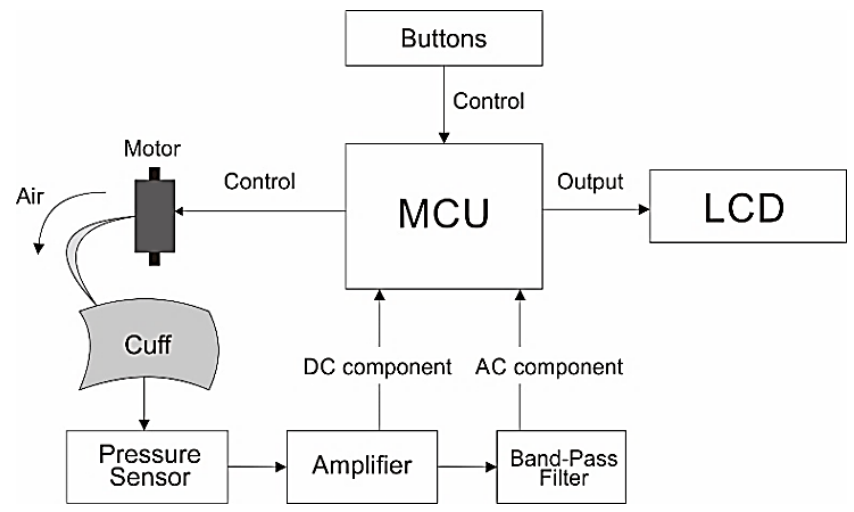

Fig. 5. Block Diagram of a Blood Pressure Sensor.

\section{Pass Band Filter Circuit}

Circuits that are built using Op-amps (Operational Amplifiers) and Capacitor and Resistor circuits serve to pass only high signals or only low ones or The Lower Frequency cutoff and the Higher Frequency cutoff [Fig. 8]. Result of 1st 
filter for Lower Frequency cutoff is $0.278 \mathrm{~Hz}$ and the result of the 1st filter is higher. The frequency cutoff is $5.837 \mathrm{~Hz}$. While the Result of 2 nd filter for Lower Frequency cutoff is $0.278 \mathrm{~Hz}$ and the result of the 2nd filter is higher. Frequency cutoff is $21.80 \mathrm{~Hz}$. whereas for the value of the mid-band gain of the first filter (A) is $-12,156$ and the mid-band gain of the Second filter (A) is 32,549 .

For the $\mathrm{Hz}$ result change the value to International Standar (SI), e.g, $56 \mathrm{uF}$ equal $56 \times 10^{-3} \mathrm{~F}$, and $10.2 \mathrm{~K}$ Resistor equal $10200 \mathrm{ohm}$.Pi value is $22 / 7$ or 3.14 , then 2 pi is 6.28 .

- The Low Bandpass 1st Filter $=$ flow $=1 /(2 \mathrm{pi}$ (C4)(R3))

- The Low Bandpass 2ndFilter $=$ flow $=1 /(2$ pi $(\mathrm{C} 3)(\mathrm{R} 1))$

- The High Bandpass 1st Filter $=$ fhigh $=1 /(2 \mathrm{pi}$ $(\mathrm{R} 4)(\mathrm{C} 2))$

- The High Bandpass 2ndFilter = fhigh $=1 /$ (2 pi $(\mathrm{R} 2)(\mathrm{C} 1))$

- $\quad$ The mid-band gain of the first filter $(\mathrm{A})=-\mathrm{R} 4 / \mathrm{R} 3$

- The mid-band gain of the Second filter $(\mathrm{A})=-\mathrm{R} 2 / \mathrm{R} 1$

\section{E. Zigbee RF Module}

In Fig. 9 shows 802.15.4 architecture, this shows there are 2 Layers on 802.15.4 architecture i.e, Zigbee and 802.15.4, in fact, the setting of ZigBee module, consist of two types ie, Zigbee $\mathrm{S} 1$ and Zigbee S2, Zigbee $\mathrm{S} 1$ is a type of Zigbee module with the function of start communication ability and Zigbee S2 to a tree and mesh communication. Therefore Zigbee S1 is IEEE 802.15.4 protocol and Zigbee S2 is Zigbee with the dynamic communication.

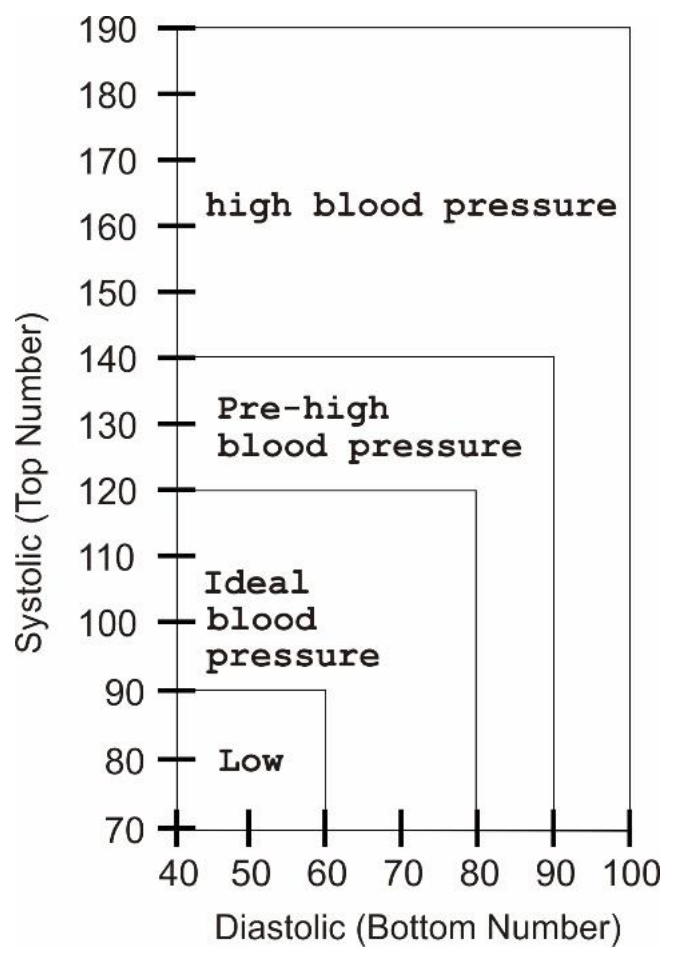

Fig. 6. Blood Pressure Classification for Adult.

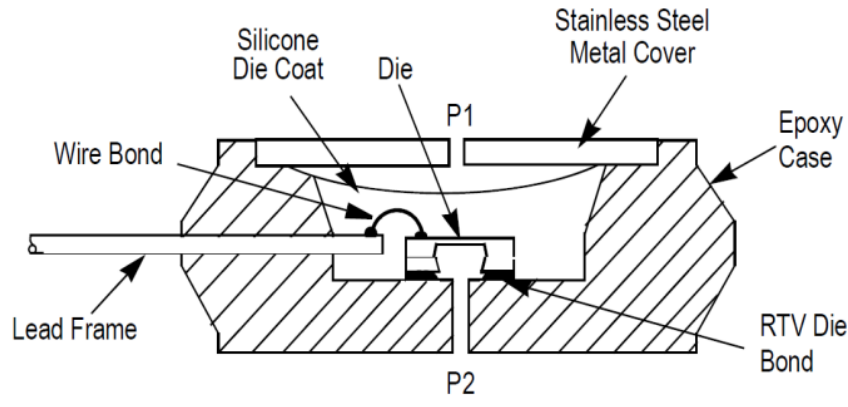

Fig. 7. Uni Body of Blood Pressure Sensor MPX10 Type.

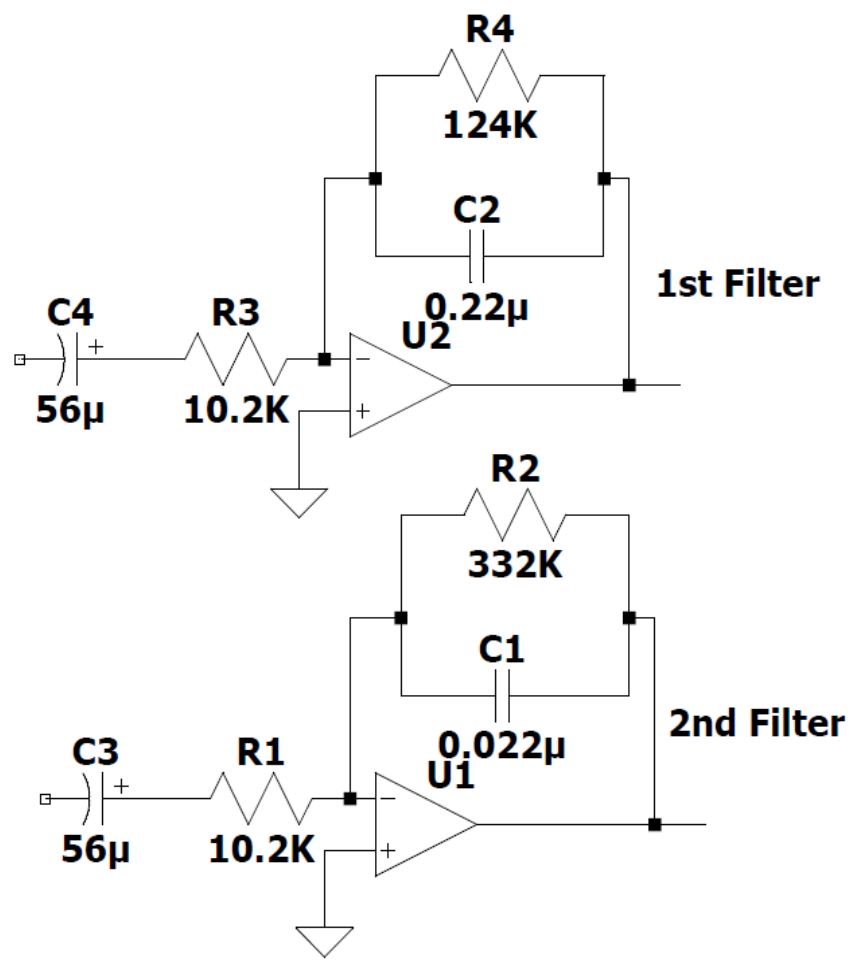

Fig. 8. Bandpass Filter Stage.

\subsection{4 architecture}

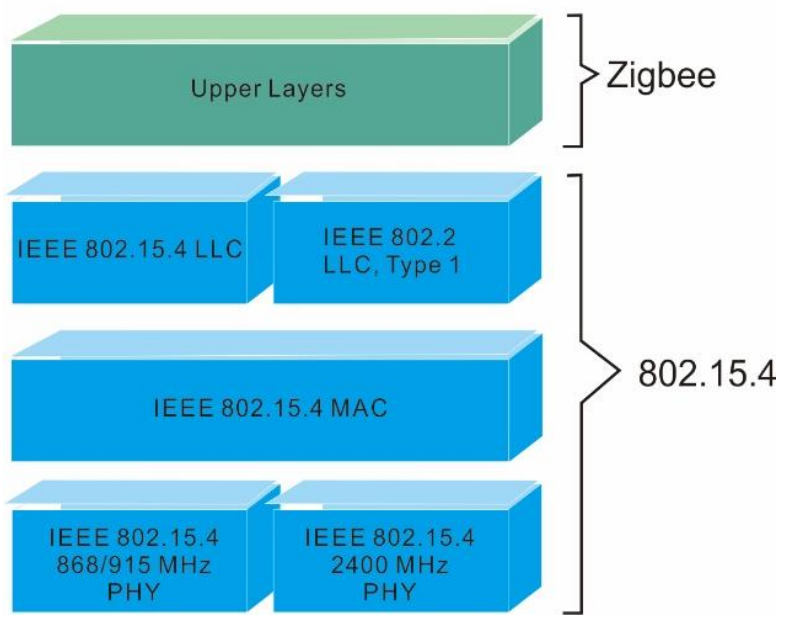

Fig. 9. Zigbee Architecture. 

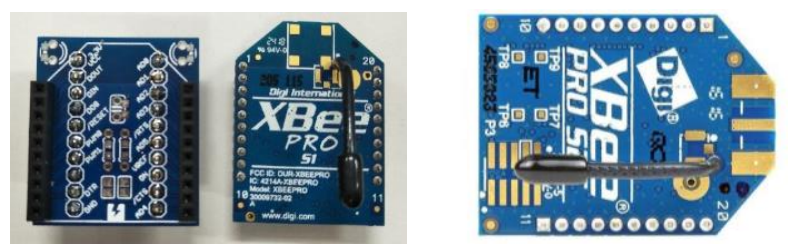

Fig. 10. Zigbee RF Module.

Zigbee is a wireless device, often referred to as a Wireless Sensor Network device, its specifications are small and Low Power and Low data rate. nevertheless, it is compatible and suitable for handling sensor Nodes, ZigBee has transmitted Power of $1 \mathrm{~mW}(0 \mathrm{dBm})$ with a data rate of $250 \mathrm{kbps}$, receiver sensitivity up to $-92 \mathrm{dBm}(1 \%$ packet error rate) and $100 \mathrm{dBm}$ (1\% packet error rate).[21] furthermore, ZigBee has a type i.e, XBee S1, Xbee S2, XBee Pro S1, and XBee Pro S2, with this specification XBee can send data in a tree, star or mesh depending on this type, S2 type can communicate between ZigBee with tree or mesh types. In this report XBee Pro S1 is used because the communication is a star network, in Fig.10 shows the ZigBee RF Module XBee Pro S1 device used in this research.

\section{F. Power Receiver (Prx (dBi)) and RSSI (dBm)}

ZigBee is Radio Frequency that has a Power Transmitter of $1 \mathrm{~mW}$ RF Power, so that if it is converted to $\mathrm{dBm}$ to $1 \mathrm{~mW}$ equal $0 \mathrm{dBm}$ equal to $-30 \mathrm{~dB}$. So when we look for the Received Signal Strength Indicator (RSSI) first determine the value of the Power Receiver (Prx) in dBi units, accordingly the theory, ZigBee is an isotropic antenna type or omnidirectional antenna whose transmitter gain value (Gtx) and receiver gain (Grx) are $-3 \mathrm{dBi}$ or $-3 \mathrm{~dB}$ (decibel). As equation (1) is the equation to determine the value of the Received Signal Strength Indicator (RSSI) in units of $\mathrm{dBm}$.

$\operatorname{RSSI}(\mathrm{dBm})=10 \log (\operatorname{Prx})$

and to determine the value of the Power Receiver (Prx), it is necessary to know the values of the Transmitter Gain (Gtx) in $\mathrm{dB}$, Gain Receiver (Grx) in $\mathrm{dB}$, Power Transmitter (Ptx) in $\mathrm{dBi}$.

While the value of the wavelength $\lambda$ is the result of the division of the speed value of light $3 \times 108$ with the value of the magnitude of the ZigBee is $2.4 \mathrm{GHz}$ frequency or equal to $24 \times 108 \mathrm{~Hz}$ so the result is $0.125 \mathrm{~m}$. accordingly, equation 2 will determine the value of the Power Receiver (dBi).

$\operatorname{Prx}(\mathrm{dBi})=\frac{P t x \cdot G t \cdot G r \cdot \lambda^{2}}{(4 \pi R)^{2}}$

$\mathrm{R}$ states the distance in units (m), with the value $\pi$ is 3.14 in short if we enter the values to find RSSI (dBm) at R or $3 \mathrm{~m}$ distance in the calculation equation [1] produces the value Prx (dB) equal $29.7 \times 10^{-3} \mathrm{~dB}$ and if included in the equation (2), the result is $R S S I=10 \log (\operatorname{Prx})$ then $10 \log \left(29.7 \times 10^{-3}\right)$, and the result is $-25 \mathrm{dBm}$. furthermore, on the results of evaluation and analysis in this research, the results will be compared between measurements in experiments and simulations with a distance of $1-00 \mathrm{~m}$ in the free space.

The RSSI relationship with d (distance) can be represented in equation (3) and equation (4). The RSSI value on the
Wireless Sensor Network of Zigbee module can be obtained with several models, one of which is the calculation of RSSI in the Free space propagation model.

RSSI $(\mathrm{d})=\operatorname{Pt}(\mathrm{D} 0)-10 \mathrm{n}_{\mathrm{p}} \log (\mathrm{D} / \mathrm{D} 0)$

Where RSSI $(d)$ is the value of RSSI in $\mathrm{dBm}$ at distance D (meters), np is the Path Loss exponent, $\mathrm{Pt}(\mathrm{D})$ is a strength of a transmitter in $\mathrm{dBm}, \mathrm{D} 0$ is a $\mathrm{D}$ at the beginning of the transmitter $\mathrm{Pt}$ (D0) at a distance of 1 meter.

$\mathrm{D}=10^{\left[\left(\mathrm{P} 0-\mathrm{Fm}-\mathrm{Pr}-10 * \mathrm{np} * \log \_10(\mathrm{f})+30 * \mathrm{np}-32.44\right) / 10 * \mathrm{np}\right]}$

Where $D$ is distance $(m), P_{0}$ is a Power transmitter $(\mathrm{dBm})$ at 0 distance, $\operatorname{Pr}$ is a signal Receiver, $F$ is Frequency $(\mathrm{Hz}), n_{p}$ is Path Loss exponent (Table I).

TABLE I. PATH LosS EXPONENT (NP)

\begin{tabular}{|l|l|}
\hline Environment & Path Loss Exponent $\left(\boldsymbol{n}_{\boldsymbol{p}}\right)$ \\
\hline Free Space & 2 \\
\hline Urban area cellular radio & 2.7 to 3.5 \\
\hline Shadowed urban cellular radio & 3 to 5 \\
\hline In Building Line-of-site & 1.6 to 1.8 \\
\hline Obstructed in building & 4 to 6 \\
\hline \multicolumn{1}{|c|}{ Obstructed in factories } & 2 to 3 \\
\hline
\end{tabular}

\section{G. Blood Pressure Node Sensor Test}

In Fig. 11 is the process of testing Blood Pressure sensors using the ATmega 328p Microcontroller, this examination was successfully carried out by storing Systolic and Diastolic sensor data $(\mathrm{mmHg})$ in the MySQL Database. furthermore, the blood pressure data processed by the ATmega $328 \mathrm{p}$ Microcontroller. furthermore, a ZigBee sends the data to the ZigBee Coordinator on the Raspberry Pi 3. The connection between the Raspberry Pi 3 and Zigbee RF module is shown in Fig. 12.

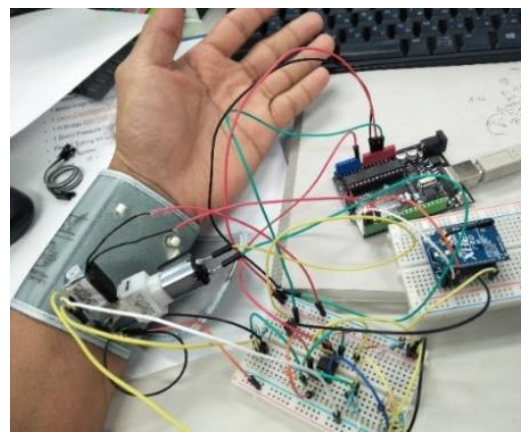

Fig. 11. Blood Pressure Connectivity Testing.

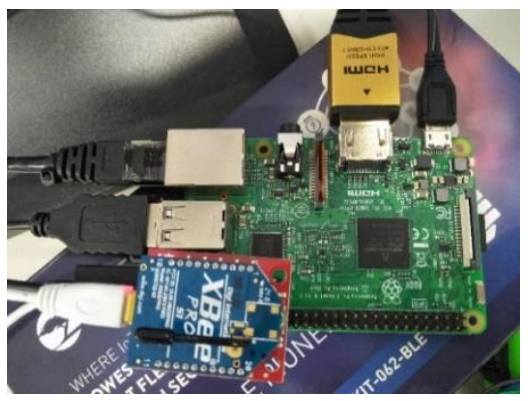

Fig. 12. Raspberry Pi 3 and Zigbee RF Module Connectivity. 


\section{H. Blood Pressure Sensor Pseudocode-1}

Pseudocode-1 shows how the Blood Pressure Sensor can work and provide Systolic and Diastolic $(\mathrm{mmHg})$ values in the Blood Pressure sensor node made in this research. In the serial output monitor, the MAP (Mean Arterial Pressure) value is different, furthermore, this is the output produced at the sensor node, i.e, the Blood Pressure value in $\mathrm{mmHg}$.

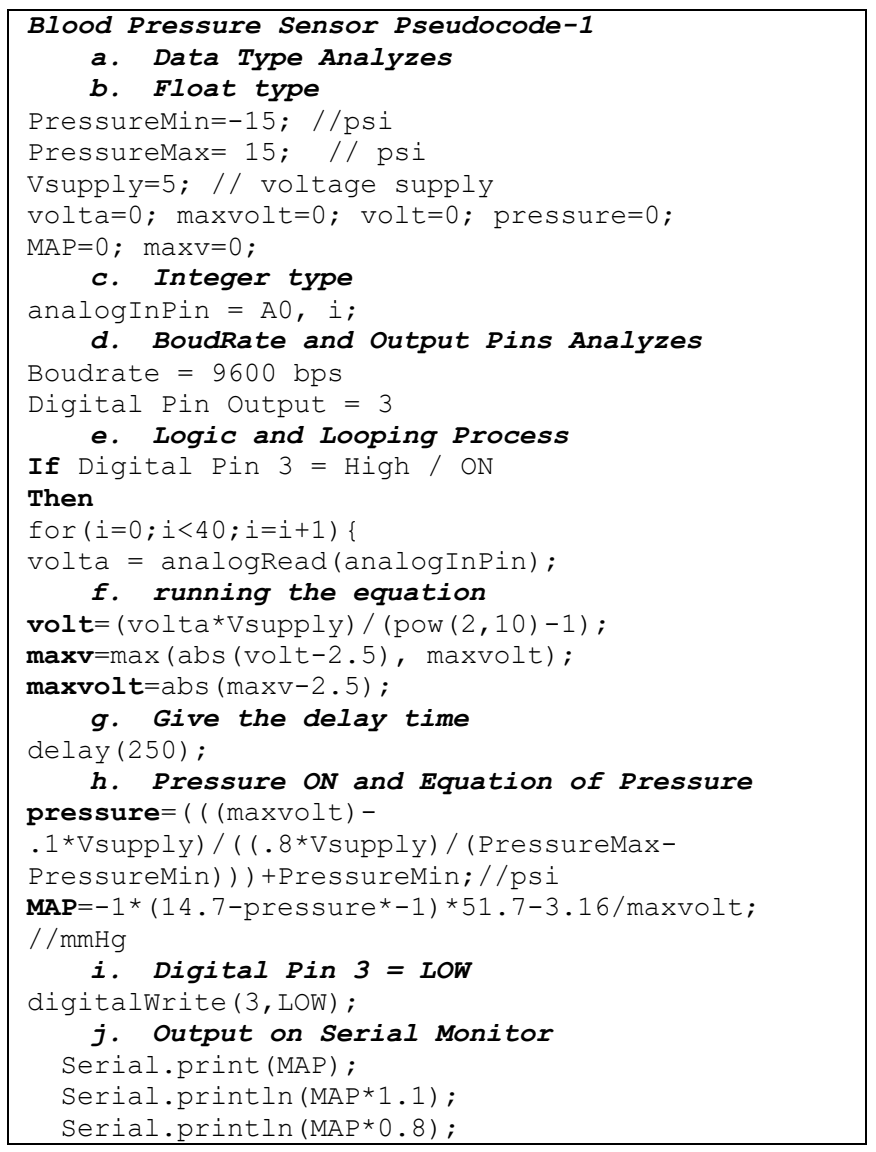

\section{ZigBee Blood Pressure in Python Pseudocode-2}

Whereas in Pseudocode-2, the input process is from Python Code to MySQL database, accordingly, input data comes from Serial Port port = '/ dev / ttyUSB0' and this is the value of the Pressure Sensor node captured by the ZigBee Coordinator Node.

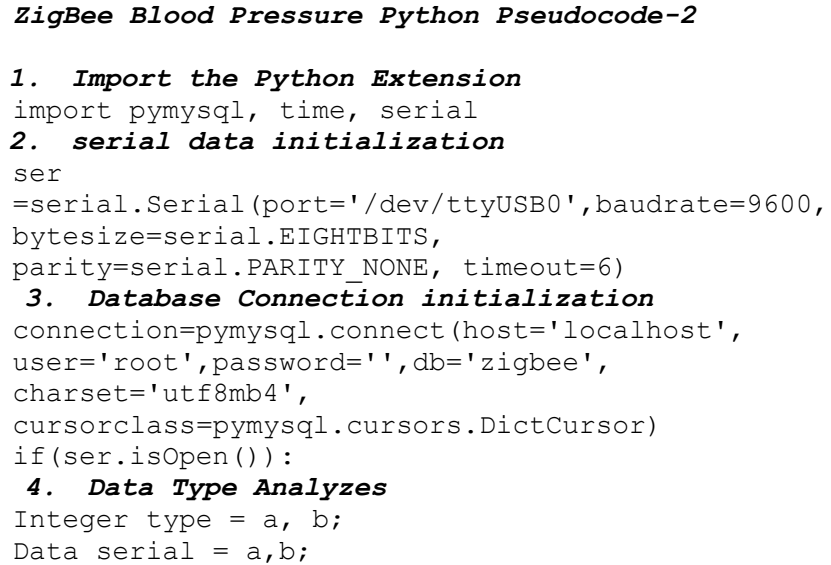

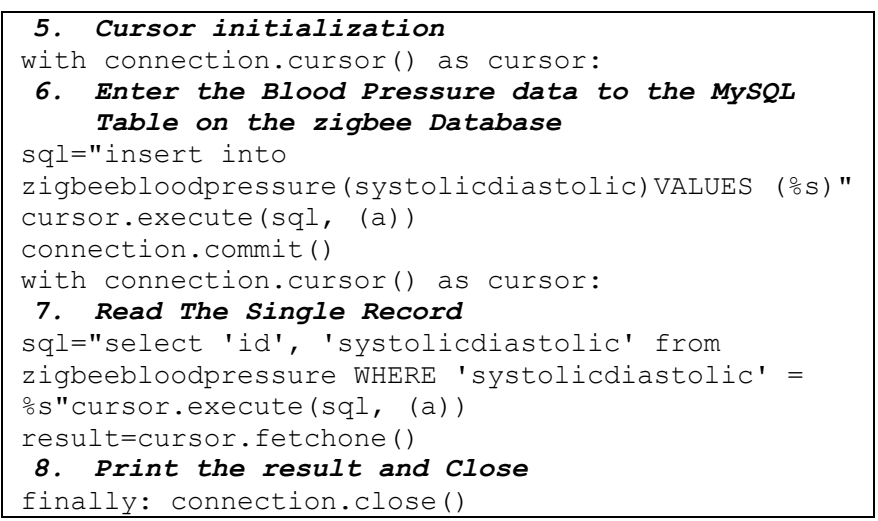

\section{J. Javascript Object Notation (JSON) Pseudocode-3}

In Pseudocode-3, Javascript Object Notation (JSON) will form a Chart that can be displayed on Web Page based on HTML and PHP, this data comes from the MySQL database, i.e, Blood Pressure Sensor data.

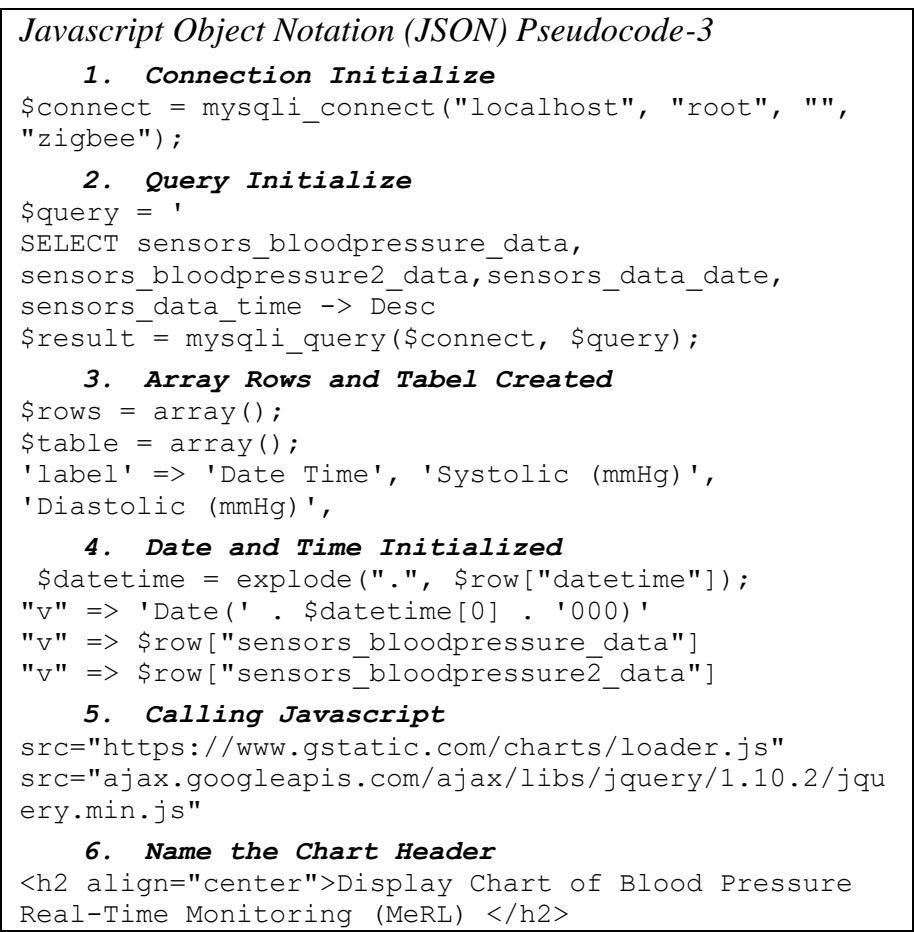

\section{RESULT AND ANALYSIS}

\section{A. Blood Pressure Sensor Testing}

Experiments a, b, c, and d [Fig. 13] are giving different treatments to the Pressure sensor. It is noted that Pulse sensors work or are active, in experiments a. a straight line on the value of Systolic and diastolic $(\mathrm{mmHg})$ is the condition when the mini-Pump is off. In experiment $b$. the graph shows an increase at one time, not every time, only 1-time increase, this is because there is no supply voltage from the amplifier IC or amplifier. accordingly, In experiment c. sensors such as losing a stable position, this is because of the Pressure sensor no supply input from a Microcontroller or Arduino Analog Pin (A0). Experiment d is a graphical difference when the sensor pressure is turned off or the mini-pump off is then turned on after a few seconds, consequently, that there is a trigger when 
the Pressure sensor is on or the mini-pump is on. The blue line shows the value of Systolic $(\mathrm{mmHg})$ and the red line shows the value of Diastolic $(\mathrm{mmHg})$, the experiment is done at the same time, the processor is the Arduino Microcontroller, and the plot or graph is formed from the Arduino Serial Port, furthermore, Arduino Microcontroller has 2 Output Functions i.e. Serial Plotting and Serial monitor, data in the form of plotting is made through the Arduino Plot series, while serial data shows the values of processing the Arduino Microcontroller in the form of Systolic and Diastolic ( $\mathrm{mmHg}$ ) values. The value in the graph shows the value of $\mathrm{mmHg}$, can be converted into an international unit, i.e, Psi, $\mathrm{kPa}$ and atm for more complex data needs.

- $1 \mathrm{~atm}=760 \mathrm{mmHg}=760$ torr $=101.3 \mathrm{KPa}=14.7 \mathrm{psi}$

- $400 \mathrm{mmHg}=400 \mathrm{mmHg} \times(1 \mathrm{~atm} / 760 \mathrm{mmHg})=0.52$ atm

- $400 \mathrm{mmHg}=400 \mathrm{mmHg} \times(101.3 \mathrm{kPa} / 760 \mathrm{mmHg})=$ $53.3 \mathrm{kPa}$

\section{B. Receiver Signal Strength Indicator (RSSI dBm)) of ZigBee RF Module}

Receiver Signal Strength Indicator (RSSI) is stated in Fig. 14. There are two comparisons, i.e, RSSI Simulation and RSSI Experiment at the field, in the results of these experiments and calculations, Zigbee communication at a distance of 5 meters, RSSI simulations show a value of -29 $\mathrm{dBm}$ and the experiment shows a value of $-40 \mathrm{dBm}$, at a distance of $100 \mathrm{~m}$, RSSI shows a value of $-55 \mathrm{dBm}$ (simulation) and $-86 \mathrm{dBm}$ (experiment).
RSSI (-dBm) experiment data retrieval is using DIGI XCTU software by sending Blood Pressure data using Zigbee from different distances, and this data is recorded every meter. RSSI (dBm) in Fig. 14 shows a decrease in signal strength. The distance of (Tx) and Receiver (Rx) determine of RSSI(-dBm) value, the farther the distance Tx-Rx, the greater the value of RSSI (-dBm). The experiment was carried out from 1-50 meters in the Free Space area.

\section{Output Graph on the Web Page}

In Fig. 15 and Fig. 16 shows the Real-time display of the MySQL data database. This data is Systolic and Diastolic $(\mathrm{mmHg})$ data, then this data is used as an indicator parameter that shows the patient's condition. This data is seen on the WEB Page, the Localhost system will be upgraded to the Domain level, so that data can be seen on all platforms, not only that JSON is used so that the quality of HTML can be used on smartphone and tablet platforms with different WEB Page views on Personal Computer, in this case, is expected to be a flexible WEB Page, so that data can be viewed easily by the user. The blue line shows the value of Systolic $(\mathrm{mmHg})$ the value of the upper part of the Blood Pressure and the red line is Diastolic ( $\mathrm{mmHg}$ ) is the value of the bottom, the graph shows the ups and downs of Systolic and Diastolic values because of several checks and different results. Furthermore, the more checks are carried out, the more blood pressure data on the MySQL database and the graphs generated will be more complex and details.

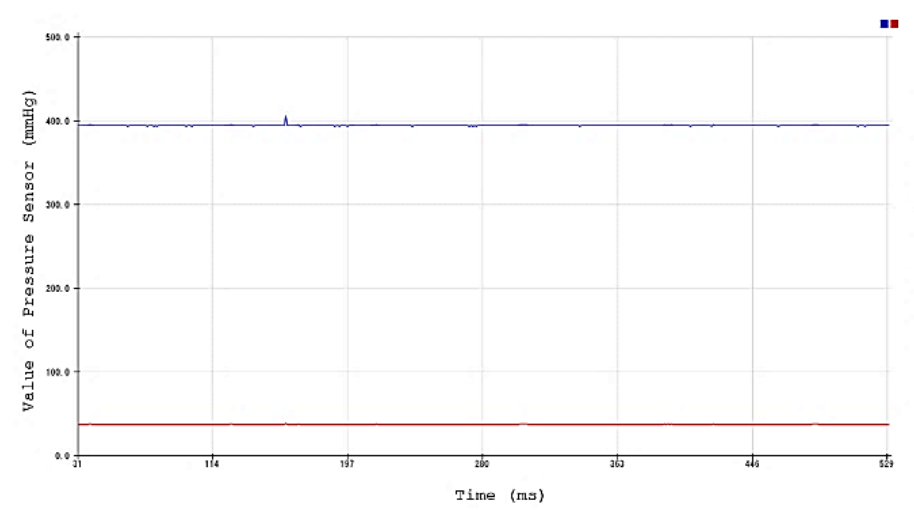

(a)

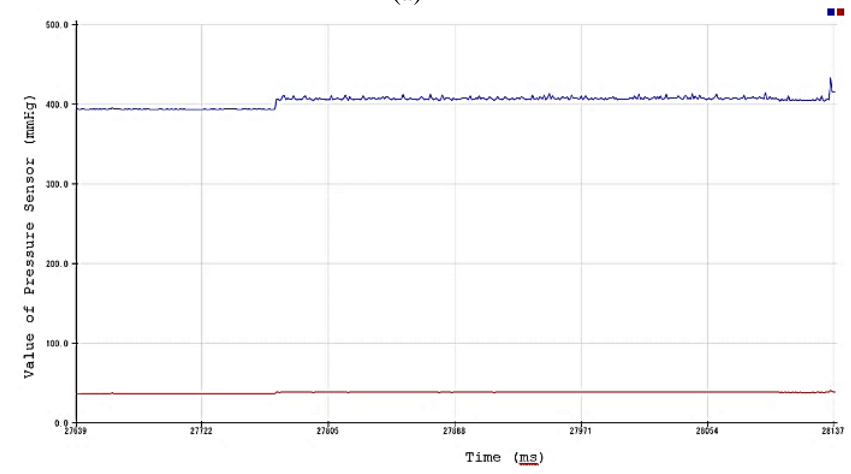

(c)

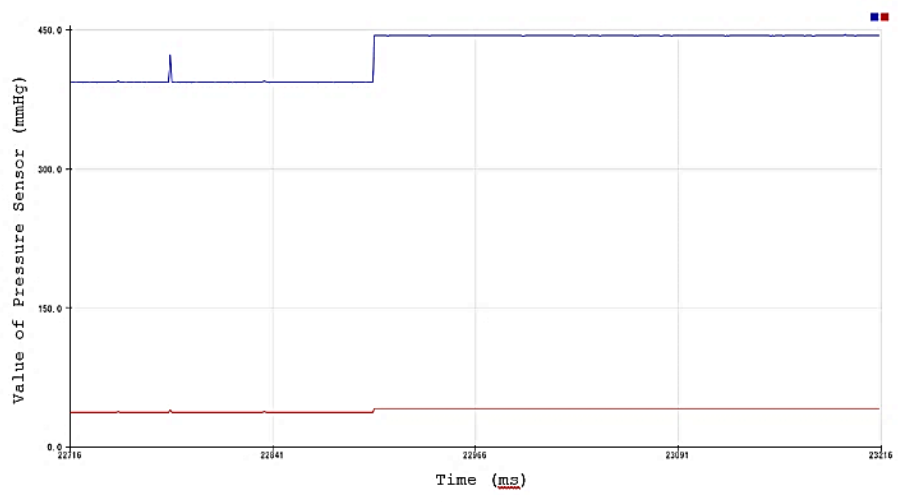

(b)

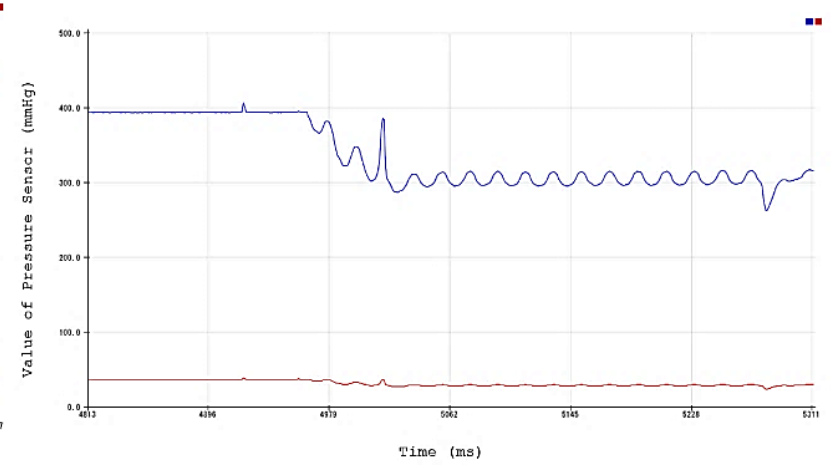

(d)

Fig. 13. Blood Pressure Test Consisting of a, b, c and d. 


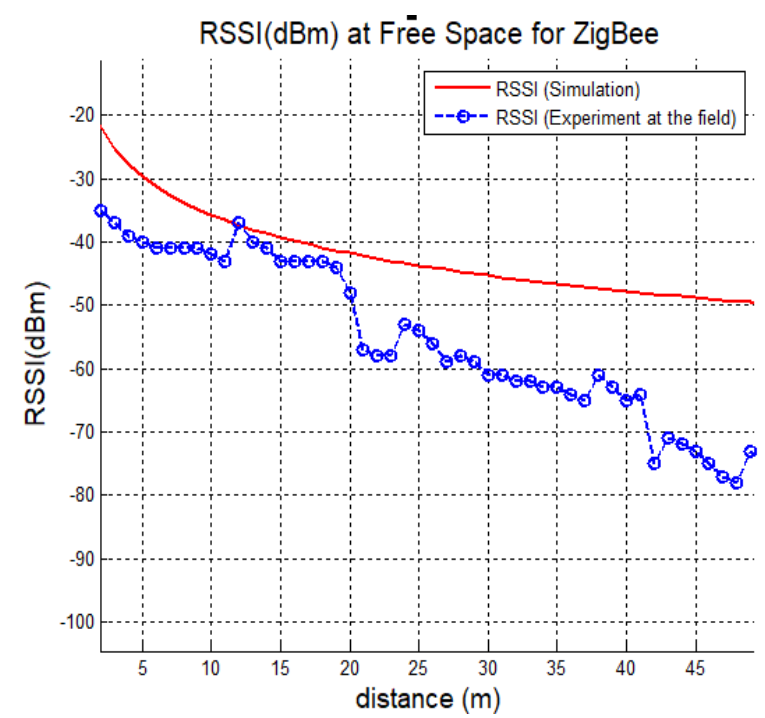

Fig. 14. RSSI (dBm) of Zigbee RF Module.

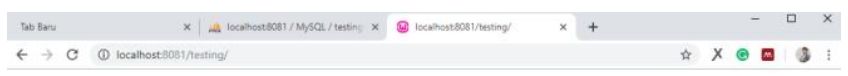

Display Chart of Blood Pressure Real-Time Monitoring (MeRL)

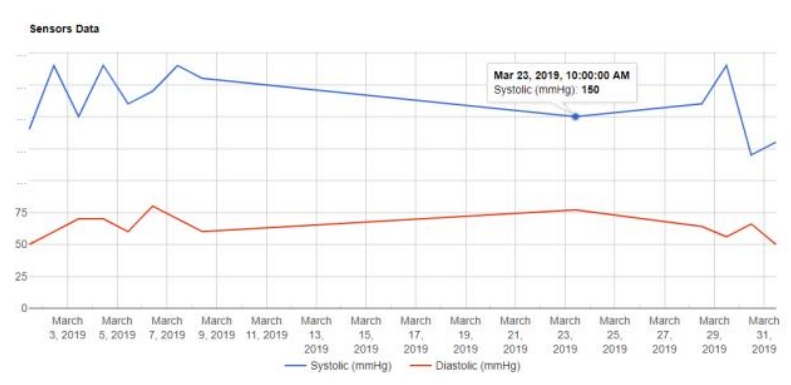

Fig. 15. Graph of Systolic (mmHg).

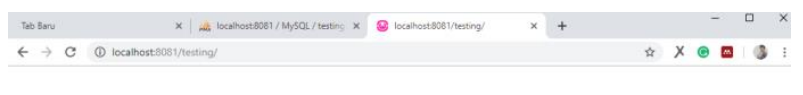

Display Chart of Blood Pressure Real-Time Monitoring (MeRL)

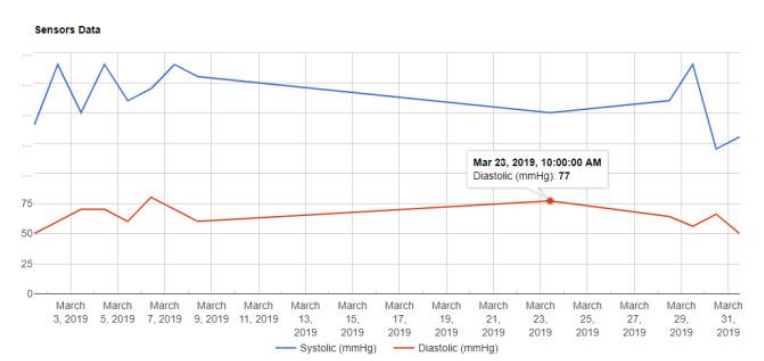

Fig. 16. Graph of Diastolic (mmHg).

\section{CONCLUSION AND SUGGESTION}

Sensor data on a ZigBee module can be sent properly as indicated by the RSSI parameter, at a distance of 5 meters RSSI simulations show a value of $-29 \mathrm{dBm}$ and $-40 \mathrm{dBm}$ in the following experiments so that it gets smaller. furthermore at a distance of $50 \mathrm{~m}$, the Receiver Signal Strength Indicator (RSSI) shows around $-70 \mathrm{dBm}$ in field measurements, while in simulations of equations 1 and 2 the RSSI results are quite good at $-50 \mathrm{dBm}$, this result is better than field measurements, at a distance $100 \mathrm{~m}$, the RSSI value produced is $-55 \mathrm{dBm}$ (simulation) and $-86 \mathrm{dBm}$ (experiment). The sensor node works very well, blood pressure can be stored in the MySQL database using the Python programming language that works on the Raspberry Pi 3 model B. And the data can be displayed on the Web Page using JSON. Accordingly, this Research still uses Localhost, it needs to be improved by moving localhost to the domain so that data can be seen on all platforms such as smartphones or tablets connected to the internet.

\section{DISCUSSION}

Some points that need to be added for future research development are (1) The prototype that is already in the form of Light type and fix on PCB and design Product, to make it comfortable for Patients (2) Programming Languages that support Graphical User Interface (GUI) output from Blood Pressure and other sensors on the mobile platform devices (3) The Security Method of IoT Protocol needs to be added to this research. Research development in monitoring patient health based on Internet of Things (IoT) is to use Algorithms for data confidentiality and security, e.g, RC4 Algorithm. RC4 Algorithm is one of the algorithms used for security on IoT using the encryption method. Improvements are also needed in this research by utilizing a programming language that supports the appearance of a capable Graphical User Interface (GUI) for display flexibility in all internet connected platforms/devices.

\section{ACKNOWLEDGMENT}

Thanks to Micro Electronics Research Laboratory (MeRL) and Professor Akio Kitagawa for providing facilities, tools, laboratories, personal computers, analyzer, hardware and software that were very useful in completing this research and also helped improve this paper so that it was better than before.

\section{REFERENCES}

[1] Muhammad Usman Ali, Soojung Hur and Yongwan Park, "Wi-Fi-Based Effortless Indoor Positioning System Using IoT Sensors", Sensors 2019, 19, 1496; doi:10.3390/s19071496

[2] Eyal Ronen, Adi Shamir, Achi-Or Weingarten, Colin O'Flynn, "IoT goes nuclear: Creating a ZigBee chain reaction", 2017 IEEE Symposium on Security and Privacy, DOI: 10.1109/SP.2017.14

[3] Puput Dani Prasetyo Adi and Akio Kitagawa, "Performance Evaluation WPAN of RN-42 Bluetooth based (802.15.1) for Sending the MultiSensor LM35 Data Temperature and RaspBerry Pi 3 Model B for the Database and Internet Gateway" International Journal of Advanced Computer Science and Applications (IJACSA), 9(12), 2018. DOI: dx.doi/10.14569/IJACSA.2018.091285

[4] Pantaleone Nespoli, David Useche Pelaez, Daniel Díaz López and Félix GómezMármol, "COSMOS: Collaborative, Seamless and Adaptive Sentinel for the Internet of Things", Sensors 2019, 19, 1492 ; doi:10.3390/s19071492

[5] Puput Dani Prasetyo Adi and Rahman Arifuddin, Design of Tsunami Detector Based Sort Message Service Using Arduino and SIM900A to GSM/GPRS Module, JEEMECS (Journal of Electrical Engineering, Mechatronic and Computer Science) Volume 1, No.1. 2018, DOI: doi/10.26905/jeemecs.v1i1.1982

[6] Ala Al-Fuqaha, Mohsen Guizani, Mehdi Mohammadi, Mohammed Aledhari, and Moussa Ayyash, "Internet of Things: A Survey on Enabling Technologies, Protocols, and Applications", IEEE Communication Surveys \& Tutorials, VOL. 17, NO. 4, Fourth Quarter 2015, DOI: 10.1109/COMST.2015.2444095 
[7] Yixin Mei, Fan Li, Lijun He and Liejun Wang, "Joint Source and Channel Rate Allocation over Noisy Channels in a Vehicle Tracking Multimedia Internet of Things System", Sensors 2018, 18, 2858; doi: $10.3390 / \mathrm{s} 18092858$

[8] Aitor Agirre, Aintzane Armentia, Elisabet Estévez and Marga Marcos, "A Component-Based Approach for Securing Indoor Home Care Applications", Sensors 2018, 18, 46; doi:10.3390/s18010046

[9] Emilio Andreozzi, Gaetano D. Gargiulo, Antonio Fratini, Daniele Esposito and Paolo Bifulco, "A Contactless Sensor for Pacemaker Pulse Detection: Design Hints and Performance Assessment", Sensors 2018, 18, 2715; doi:10.3390/s18082715

[10] Dimiter V. Dimitrov, MD, PhD, "Medical Internet of Things and Big Data in Healthcare", A Digital Achive and Reference Linking Platform of Korean Medical Journals, Published online July 31, 2016. https://doi.org/10.4258/hir.2016.22.3.156

[11] Giorgio Biagetti, Paolo Crippa, Laura Falaschetti and Claudio Turchetti, "Classifier Level Fusion of Accelerometer and sEMG Signals for Automatic Fitness Activity Diarization", Sensors 2018, 18, 2850; doi:10.3390/s 18092850

[12] Muhammad Niswar, Amil Ahmad Ilham, Elyas Palantei, Rhiza S. Sadjad, Andani Ahmad, Ansar Suyuti, Indrabayu, Zaenab Muslimin, Tadjuddin Waris, Puput Dani Prasetyo Adi, "Performance evaluation of ZigBee-based wireless sensor network for monitoring patients' pulse status”, 2013 International Conference on Information Technology and Electrical Engineering (ICITEE) DOI: doi/10.1109/ICITEED.2013 .6676255

[13] Muhammad Anwar, Abdul Hanan Abdullah, Ayman Altameem, Kashif Naseer Qureshi, Farhan Masud, Muhammad Faheem, Yue Cao, and Rupak Kharel, "Green Communication for Wireless Body Area Networks: Energy Aware Link Efficient Routing Approach", Sensors 2018, 18(10), 3237; https://doi.org/10.3390/s18103237
[14] Edith Osorio de la Rosa , Javier Vázquez Castillo , Mario Carmona Campos, Gliserio Romeli Barbosa Pool, Guillermo Becerra Nuñez, Alejandro Castillo Atoche and Jaime Ortegón Aguilar, "Plant Microbial Fuel Cells-Based Energy Harvester System for Self-powered IoT Applications", Sensors 2019, 19, 1378; doi:10.3390/s19061378

[15] Moh. Khalid Hasan, Md. Shahjalal, Mostafa Zaman Chowdhury and Yeong Min Jang, " Real-Time Healthcare Data Transmission for Remote Patient Monitoring in Patch-Based Hybrid OCC/BLE Networks ", Sensors 2019, 19, 1208; doi:10.3390/s19051208

[16] Gaël Loubet, Alexandru Takacs, Ethan Gardner, Andrea De Luca, Florin Udrea and Daniela Dragomirescu, "LoRaWAN Battery-FreeWireless Sensors Network Designed for Structural Health Monitoring in the Construction Domain", Sensors 2019, 19, 1510; doi:10.3390/s19071510

[17] José Santa, Ramon Sanchez-Iborra, Pablo Rodriguez-Rey, Luis BernalEscobedo and Antonio F. Skarmeta, "LPWAN-Based Vehicular Monitoring Platform with a Generic IP Network Interface", Sensors 2019, 19, 264; doi:10.3390/s19020264

[18] Muhammad Ateeq, Farruh Ishmanov, Muhammad Khalil Afzal, and Muhammad Naeem, "Multi-Parametric Analysis of Reliability and Energy Consumption in IoT: A Deep Learning Approach", Sensors 2019, 19, 309; doi:10.3390/s19020309

[19] Adolfo Di Serio, John Buckley, John Barton, Robert Newberry, Matthew Rodencal, Gary Dunlop 3 and Brendan O'Flynn, "Potential of Sub-GHzWireless for Future IoT Wearables and Design of Compact 915 MHz Antenna", Sensors 2018, 18, 22; doi:10.3390/s18010022

[20] Jiheon Kang and Doo-Seop Eom, "Offloading and Transmission Strategies for IoT Edge Devices and Networks", Sensors 2019, 19, 835; doi:10.3390/s19040835

[21] Puput Dani Prasetyo Adi, Analisis kinerja jaringan sensor nirkabel untuk monitoring denyut nadi pasien, April 2018, DOI: 10.13140/RG.2.2.29145.83040. 